\title{
MODELS FOR BIOLOGICAL PHOSPHORYL TRANSFER
}

\author{
NICHOLAS H. WILLIAMS \\ Centre for Chemical Biology, Krebs Institute for Biomolecular Science, \\ Department of Chemistry, Sheffield University, Sheffield, S3 7HF, UK
}

Phosphoryl transfer at the phosphate mono- and diester level is ubiquitous in biology. However, the detailed understanding of how these reactions proceed and the corresponding enzyme catalysed processes are still the subject of debate despite the extensive physical organic chemistry data that has accumulated over the years. Recent work has revealed that both processes are extremely slow under physiological conditions, and that the corresponding efficiencies of the enzymes that catalyse these reactions are the highest established to date [1,2]. This suggests that these enzymes have very high transition state affinities, and so will be very sensitive to accurate transition state mimics.

Despite the observation that alkyl phosphate mono- and diesters have comparable reactivity, the transition states for hydrolysis in solution are significantly different. Diesters are believed to react through a phosphorane intermediate or phosphorane-like ("associative") transition state. Monoesters are believed to have a "dissociative" transition state that has much less bonding to the nucleophile and leaving group.

This obviously has implications for transition state mimics and the understanding of how catalysis is facilitated. This is energetically most efficient if the mechanistic pathway in solution is stabilised; however, in many instances, it is suggested that enzymes catalyse monoester transfer through an associative (diester-like) pathway. This means that the rate acceleration has to be yet higher to account for the observed rates, but is perhaps easier to rationalise in terms of the active site residues available. For example, in the catalytic subunit of protein phosphatase 1 (PP1), these are metal ions and cationic side chains which might be expected to stabilise developing negative charge (see figure below left). This leads on to the idea that by using multiple interactions simultaneously, cooperativity in catalysis is achieved i.e. the overall effect is greater than the sum of the parts. However, this intriguing idea is difficult to demonstrate unambiguously in large, complex natural systems.

This contribution examines the background reactivity of phosphate esters, and reports data showing that the substrates for serine/threonine phosphatases have slower intrinsic rate constants than any other enzyme substrates. Using model complexes (see figure above right), the characteristics of alternative (associative) mechanisms that have been proposed for the metallophosphatase catalysed reaction are explored [3,4]. Finally, complementary catalytic groups are combined with this core complex to look for experimental evidence for possible cooperativity in this context $[5,6]$. 

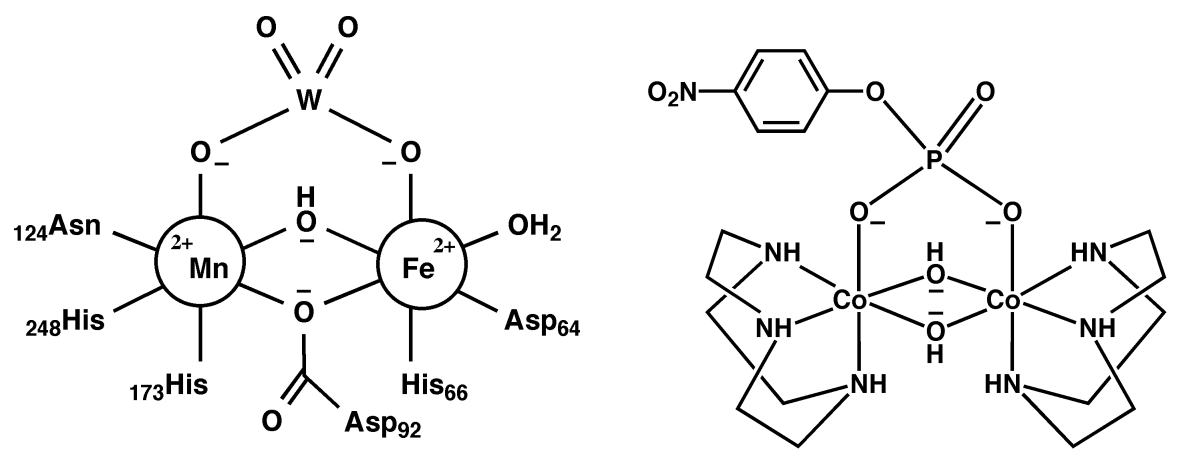

\section{REFERENCES}

1. Lad, C., Williams, N.H. and Wolfenden, R.V. Rates of spontaneous hydrolysis of phosphate ester dianions and the extreme catalytic proficiencies of phosphatases involved in cell signaling and regulation. Proc. Natl. Acad. Sci. USA 100 (2003) 5607-5610.

2. Williams, N.H. and Wyman, P. Base catalysed phosphate diester hydrolysis. Chem. Comm. 14 (2001) 1268-1269.

3. Williams, N.H., Lebuis A-M. and Chin, J. A structural and functional model of dinuclear metallophosphatases. J. Am. Chem. Soc. 121 (1999) 33413348.

4. Humphry, T., Forconi, M., Williams N.H. and Hengge A.C. An altered mechanism of hydrolysis for a metal-complexed phosphate diester. J. Am. Chem. Soc. 124 (2002) 14860-14861.

5. Williams, N.H. Magnesium (II) catalysed ATP hydrolysis of ATP. J. Am. Chem. Soc. 122 (2000) 12023-12024.

6. Forconi, M. and Williams, N.H. Mimicking metallophosphatases: Revealing a role for an $\mathrm{OH}$ group with no libido. Angewandte Chemie, Int. Ed. Engl. 41 (2002) 849-852. 